\title{
A COMPARISON OF CREATING AN ANDROID APPLICATION USER INTERFACE IN XAML AND IN C\# CODE \\ Igor Košt’ál ${ }^{1}$, Martin Mišút ${ }^{2}$
}

\begin{abstract}
Almost every Android user application has some kind of user interface. Android programmers who create Xamarin.Forms applications and who uses the Microsoft Visual Studio development environment to do so can create user interfaces in the XAML (the Extensible Application Markup Language) or in the C\# programming language. This paper deals with a comparison of creating this user interface by the first and the second way. We demonstrate the differences in the creation of Android application user interfaces in XAML and in C\# by way of using two of our Android applications which were created by the Microsoft Visual Studio 2019 Enterprise, which work as text editors with the ability to store text to disc, and have user interfaces that are visually identical. However, the user interface of the first Android application was created in XAML with a C\# support code, whereas the user interface of the second Android application was created entirely in C\#. While comparing the source codes of the user interfaces of both these Android applications, we identify the advantages and disadvantages of both approaches to creating a user interface and we try to find out which of these approaches is better for maintaining and modifying user interfaces. In this study, we also contrast the processes of handling events of controls of a user interface created in the XAML code for the first Android application as well as that of the same user interface created in the C\# code for the second Android application. Furthermore, we were interested in determining whether the different ways of creating user interfaces affected the execution time of basic operations that included disc files that were performed on the same data by both the applications. We assume that it does not fundamentally affect the execution time, and so, we performed an experiment to confirm or refute our assumption.
\end{abstract}

UDC Classification: 004.42, DOI: https://doi.org/10.12955/pns.v2.153

Keywords: Android Xamarin.Forms application, user interface, XAML, C\# code

\section{Introduction}

An Android Xamarin.Forms application programmer who uses the Microsoft Visual Studio development environment to create it can construct its user interface in XAML (the Extensible Application Markup Language) or in the C\# programming language. This allows him to use the Xamarin.Forms mobile development platform that lets developers write user-interface source codes in C\# or XAML and those can be further compiled for iOS, Android, and Windows devices. When we specify this option, the developer can choose one out of three options. He can write the source code of the Xamarin.Forms application user interface entirely in C\#, or entirely in XAML, or in a combination of the XAML and C\# code, whilst for all the three options the event handlers of the user interface controls are always written in C\# code. We were interested in determining the differences in creating the Android Xamarin.Forms application user interface in XAML and C\#, as well as in finding out what advantages and disadvantages can result for developers and software companies from either one of the choices.

We created two Android Xamarin.Forms applications in the Microsoft Visual Studio 2019 Enterprise by using the Xamarin.Forms template. These applications worked as text editors and had user interfaces that were visually identical. However, the user interface of the first application was created in XAML with a C\# support code and the user interface of the second application was created entirely in C\#. Comparatively, we identify various advantages and disadvantages for both the approaches and we try to answer the question of determining which of these approaches is better for maintaining and modifying user interfaces. We also compare the processes of handling events of controls of the same user interface created in both the XAML code and the C\# code for the Android application. We were interested to know whether the programmer had to deal with the process of handling events of controls of a user interface created in XAML. During the experiment, we also examined whether the different ways of creating the user interfaces of these Android applications affected the execution time of basic operations that involved disc files and that were performed on the same data in the applications. It is necessary to maintain information about all these aspects while deciding which approach is to be chosen. If it is possible to define all the objects of the Android application user interface controls together with the configurations of their properties in the XAML code, we would only be required to write event handlers for these controls in the C\# code. Then, for large Xamarin.Forms projects, software companies could

\footnotetext{
${ }^{1}$ University of Economics in Bratislava, Faculty of Economic Informatics, Department of Applied Informatics, Bratislava, Slovakia, igor.kostal@euba.sk

${ }^{2}$ University of Economics in Bratislava, Faculty of Economic Informatics, Department of Applied Informatics, Bratislava, Slovakia, martin.misut@euba.sk
} 
split this work on creating user interfaces of applications between designers and programmers. Designers would only need to design a user-friendly design of the Xamarin.Forms application user interface and programmers would only need to write event handlers for the interface controls and the remaining logic of such an application in C\#. If our hypothesis is confirmed, the creation of the Xamarin.Forms application user interface in XAML and the event handlers for the interface controls in C\# will allow a software company to create Xamarin.Forms applications with an attractive user interface and a running flawless, efficient, and powerful application core.

Using XAML and C\# Code for Creating an Android Xamarin.Forms Application User Interface

We can write entire Xamarin.Forms applications in C\# (Petzold, 2016). However, we can write some parts of Xamarin.Forms applications, for example, their user interfaces, in an alternative XAML (the Extensible Application Markup Language) provided by Xamarin.Forms.

XAML is a declarative markup language used for instantiating and initializing objects (Microsoft, 2021). We can use XAML for defining tree-structured visual user interfaces characteristic of graphical programming environments (Petzold, 2016). "The XAML implementation in Xamarin.Forms supports the visual elements defined by Xamarin.Forms, such as Label, BoxView, Frame, Button, StackLayout, and ContentPage" (Petzold, 2016), which we can use for creating a Xamarin.Forms application user interface control.

When we write the entire Xamarin.Forms application code in C\#, then we usually "define the initial appearance of its user interface in the constructor of a class that derives from the ContentPage class" (Petzold, 2016). If we choose to use XAML, the markup generally replaces this constructor code (Petzold, 2016). We further find that $X A M L$ provides a more succinct and elegant definition of the user interface and has a visual structure that better mimics the tree organization of the visual elements on the page (Petzold, 2016). XAML is also generally easier to maintain and modify, and is more concise than its equivalent $\mathrm{C \#}$ code (Petzold, 2016).

But, XAML has several deficiencies too that are intrinsic to markup languages: XAML has no loops, no flow control, no algebraic calculation syntax, and no event handlers. However, using XAML has several distinct advantages that can compensate for some of these deficiencies.

We are more inclined to know whether the mentioned advantages of using XAML for creating an Android application user interface will be demonstrated while analyzing the source codes of the two of our Android application user interfaces as opposed to the use of the C\# language.

\section{Two Android Xamarin.Forms Applications with Visually Identical User Interfaces, but Created in Different Ways}

We created two Android Xamarin.Forms applications, the EditorWithXAML and the EditorWithoutXAML, with the help of the Microsoft Visual Studio 2019 Enterprise by using the same Xamarin.Forms template. It, in turn, created three projects: one common project (the Portable Class Library project), and two platform projects - for Android and for iOS. Here, we only deal with Android applications and projects related to them. The Xamarin.Forms template prefers using XAML to create the application user interface. Therefore, the common projects of both applications contain XAML files. It is due to using the same template to create solutions for both applications that the common projects of both solutions contain source files with the same name, but with different content. These Android applications work as text editors and have user interfaces that are visually identical. Nevertheless, the user interface of the EditorWithXAML application was created in XAML with a C\# support code, and the user interface of the EditorWithoutXAML application was created entirely in C\#.

The source codes of the common projects for both Android Xamarin.Forms applications are divided into four parts which are then saved into files:

- App.xaml - this XAML file contains an $x$ :Class specification which indicates that the App class in the EditorWithXAML or EditorWithoutXAML namespaces derives from the Application class.

- App.xaml.cs - this is the code-behind file for the App.xaml file. The App.xaml.cs file contains the App class definition.

- MainPagexaml - this XAML file contains the definition of our EditorWithXAML Android application's tree-structured user interface that was created in XAML (Figure 2a). Objects of 
all controls are instantiating and initializing in a XAML code of this file. This XAML file for the EditorWithoutXAML Android application only contains the empty ContentPage element.

- MainPage.xaml.cs — this is the code-behind file for the MainPage.xaml file. The MainPage.xaml.cs file contains a code that supports the markup and creates the underlying logic of the user interface for our EditorWithXAML Android application and the full logic of the entire EditorWithoutXAML Android application (Figure 2b).

The key class in the source codes of both applications is the MainPage class which derives from the ContentPage class. Its definition is split into MainPage.xaml and MainPage.xaml.cs source files in both applications.

The MainPage class of the EditorWithXAML application contains the following members:

- The MainPage constructor

- The EditorTextChanged, OnFileListViewItemSelected, OnDeleteTextButtonClicked, OnDisplay PathButtonClicked, OnSaveButtonClicked, OnDeleteButtonClicked, and OnNewFileButtonClicked methods - these are event handlers for the application's user interface controls

- The RefreshListView method - reloads all items of the fileListView object of the ListView class

- The GetFiles method - obtains the current filenames from an application local storage

- The labelPathFileName, editor, fileListView, deleteTextBtn, saveFileBtn, deleteFileBtn, and newFileBtn fields - the objects of the controls of the application's user interface

- The fileName8v15e, fileName10v17e, fileName_userG, fileName_LogF, selectedItem_str, and docsPath fields - these fields use some event handlers and other methods

Figure 1 shows all members of the MainPage and App classes of the EditorWithXAML application.

The MainPage class of the EditorWithoutXAML application contains similar and rather identical members as the MainPage class of the EditorWithXAML application:

- The MainPage constructor

- The Editor_TextChanged, FileListView_ItemSelected, DeleteTextbtn_Clicked, DisplayPathbtn_Clicked, SaveFileBtn_Clicked, DeleteFileBtn_Clicked, and NewFileBtn_Clicked methods - these are event handlers for the application's user interface controls

- The RefreshListView method - reloads all items of the fileListView object of the ListView class

- The GetFiles method - obtains the current filenames from an application local storage

- The labelPathFileName, editor, fileListView, saveFileBtn, deleteFileBtn, and newFileBtn fields - the objects of the controls of the application's user interface

- The fileName_app1, fileName_app2, fileName_user, fileName_LogF, selectedItem_str, and docsPath fields - these fields use some event handlers and other methods

The first object that we instantiate in XAML code of the EditorWithXAML application user interface is the object of the StackLayout class, which arranges its children in a stack. This StackLayout object is expressed as a StackLayout XML element in the XAML code of the MainPage.xaml source file. The StackLayout XML element contains three child XML elements, the Label, the Editor, and the Grid. These child elements represent objects of the Label, the Editor, and the Grid classes. The Margin attribute of the StackLayout XML element sets the inner padding of the layout.

The Text attribute of the Label child element sets the text for the label, the HorizontalOptions attribute specifies how the label is to be positioned relative to its parent (in this case the StackLayout object) and the FontAttributes attribute sets the font style of the text for this label.

The TextChanged attribute of the Editor child element attaches the EditorTextChanged event handler to the TextChanged event of this element. This event is raised when the text of the editor changes. The EditorTextChanged event handler resides in the MainPage.xaml.cs code-behind file.

The Placeholder attribute of the Editor child element sets the text that is displayed when the control is empty and the HeightRequest attribute sets the desired height override for this element.

The x:Name attribute of the Label and Editor child elements gives specific names to objects of the Label and Editor classes. 


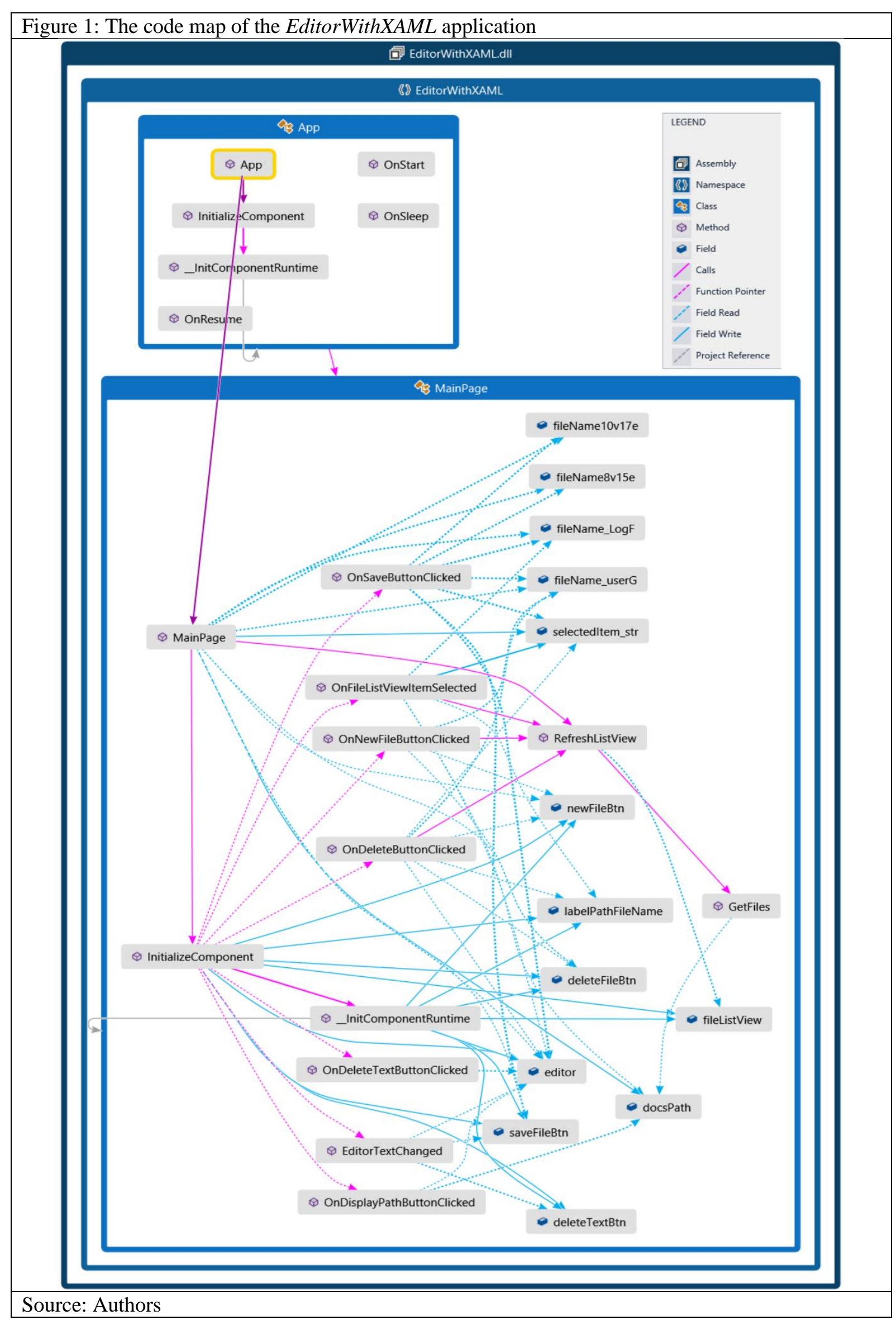




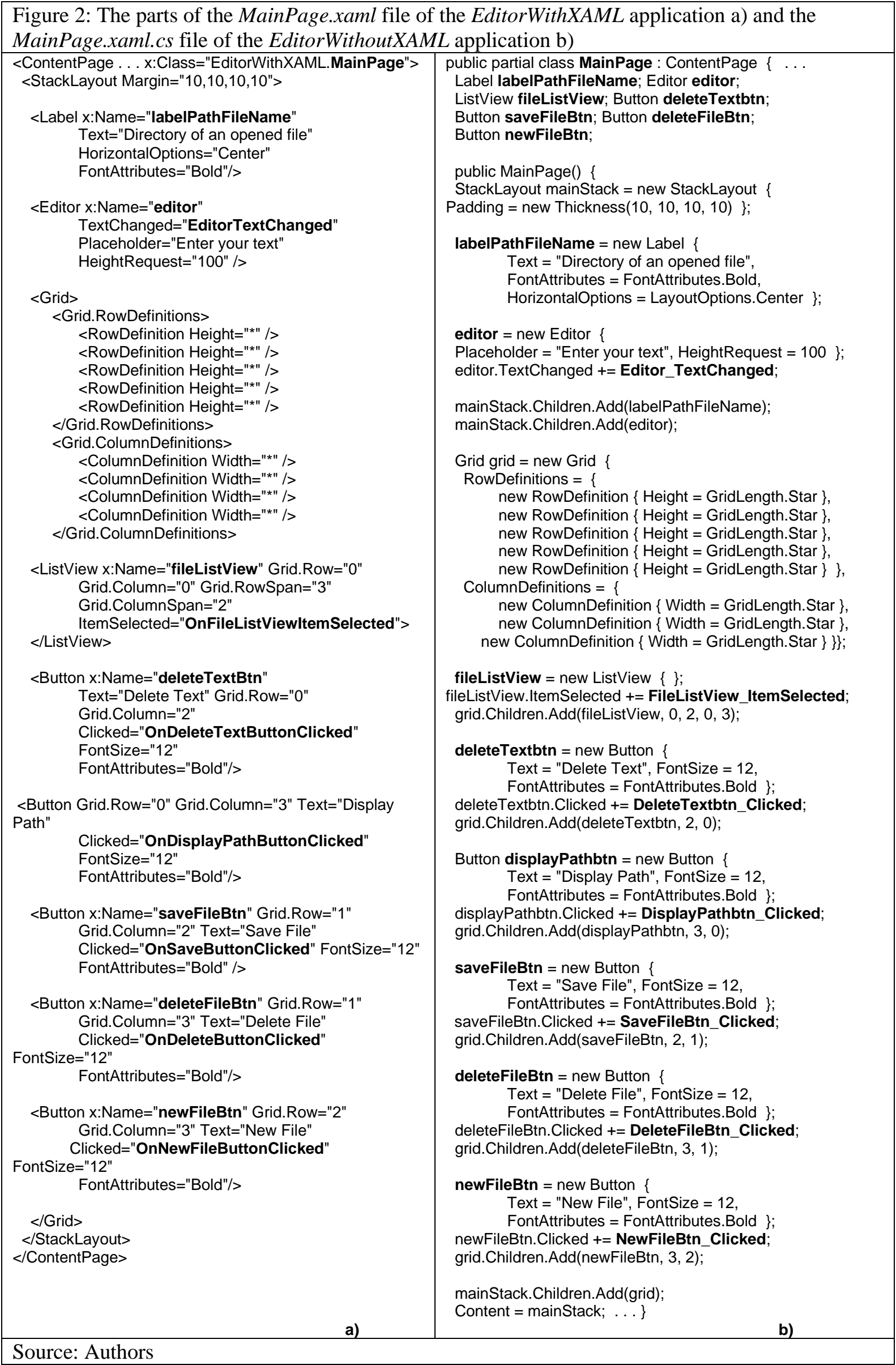


We have used objects of the Grid class to lay out additional controls in both the Android applications' user interfaces. This Grid class provides a powerful layout mechanism that organizes its children into rows and columns of cells (Petzold, 2016). The Grid class is designed specifically for two-dimensional arrays of children and it can also be very useful for managing layouts that adapt to both portrait and landscape modes (Petzold, 2016). A Grid object can be defined and filled with children in either C\# code or XAML, but the XAML approach is easier and clearer, and hence, by far, the more common one (Petzold, 2016).

The Grid object definition of the EditorWithXAML application contains definitions of two important properties — the RowDefinitions and ColumnDefinitions. The RowDefinitions property defines a collection of RowDefinition objects; one RowDefinition object for every row in the Grid object. The ColumnDefinitions property defines a collection of ColumnDefinition objects; one ColumnDefinition object for every column in the Grid object. Both these collections define the row and column characteristics of the Grid object (Petzold, 2016). The Grid objects of the EditorWithXAML and EditorWithoutXAML applications have five rows and four columns. All RowDefinition objects of both Grid objects set the Height equal to "** or GridLength.Star and all ColumnDefinition objects of both Grid objects set the Width equal to "*" or GridLength.Star, which means that the height and width of both the Grid objects' areas are divided equally between the five rows and the four columns.

To specify the height of RowDefinition objects and the width of the ColumnDefinition objects in the EditorWithoutXAML application's grid object, we use the Star value of the GridLength structure.

The Grid object of the EditorWithXAML application is expressed as the Grid XML element in the XAML code of the MainPage.xaml source file. The Grid XML element contains six child XML elements, the ListView, and five Button elements. These child elements represent one object of the ListView class and five objects of the Button class. The Grid.Row and Grid.Column attributes of these child elements specify cells of the Grid object which these elements occupy. The Grid.RowSpan and Grid.ColumnSpan attributes of the ListView child element specify that this element occupies the two rows of the first column and the two columns of the first row.

The ItemSelected attribute of the ListView child element attaches the OnFileListViewItemSelected event handler to an ItemSelected event. When a user selects some item in the ListView control, it fires an ItemSelected event. The OnFileListViewItemSelected event handler itself resides in the MainPage.xaml.cs code-behind file.

The Clicked attribute of every Button child element attaches a given event handler to a Clicked event of a given child element. When a user taps a Button control, it fires a Clicked event (Petzold, 2016). Event handlers of all Button child elements reside in the MainPage.xaml.cs code-behind file.

The Text attribute of every Button child element sets the text to display the content of a given button.

The FontSize and FontAttributes attributes of every Button child element set the size of the font of a given button text and the font style of this button text.

The $x$ :Name attribute of the ListView child element and four Button child elements gives specific names to objects of the ListView and Button classes.

As we mentioned above in a description of the XAML code of the EditorWithXAML application user interface elements, event handlers of the user interface controls, which represent these elements, are written in C\# code saved into the MainPage.xaml.cs code-behind file.

All that we did in the XAML code of our EditorWithXAML application (Figure 2a) is also described in detail above. We did the same in the second EditorWithoutXAML application in the C\# code (Figure $2 b)$.

We created the mainStack, grid objects and the labelPathFileName, editor, fileListView, deleteTextbtn, displayPathbtn, saveFileBtn, deleteFileBtn, newFileBtn objects of all controls of the EditorWithoutXAML application user interface using constructors of the StackLayout, Grid, Label, Editor, ListView and Button classes. We set and specified all properties of these objects within these constructors. We attached event handlers to particular events of all the controls by EventHandler delegates. We also used Add methods for adding children to the Children collections of the mainStack and grid objects. 
For example, here we create a new editor object by using the constructor of the Editor class, setting its two Placeholder and HeightRequest properties within the constructor, attaching the EditorTextChanged event handler to the TextChanged event of this object, and by adding the editor child to the Children collection of the mainStack object.

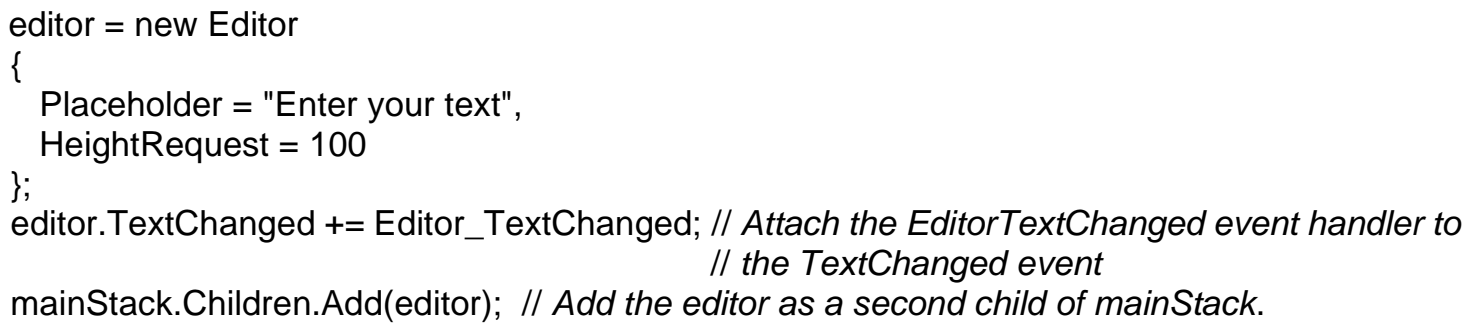

Other constructors and other codes of the main MainPage constructor are displayed in Figure 2b).

As observed, it is feasible to define all objects of the Android application user interface controls "in C\# code, but usually without the clarity or orderliness of the XAML definition" (Petzold, 2016).

When we compare the XAML code with the C\# code for the two of our Android Xamarin.Forms application user interfaces, which have the same visual appearance, we can clearly see that the XAML code "provides a more succinct and elegant definition of the user interface and has a visual structure that better mimics the tree organization of the visual elements on the page" (Petzold, 2016).

Also, as is evident, it is fairly viable to define all objects of the Android application user interface controls together with configurations of their properties in the XAML code, and we would only be required to write the event handlers for these controls in the $\mathrm{C \#}$ code. The software company could then divide the work on creating Android Xamarin.Forms application user interfaces between designers and programmers. This confirms the hypothesis that we formulated towards the end of the Introduction.

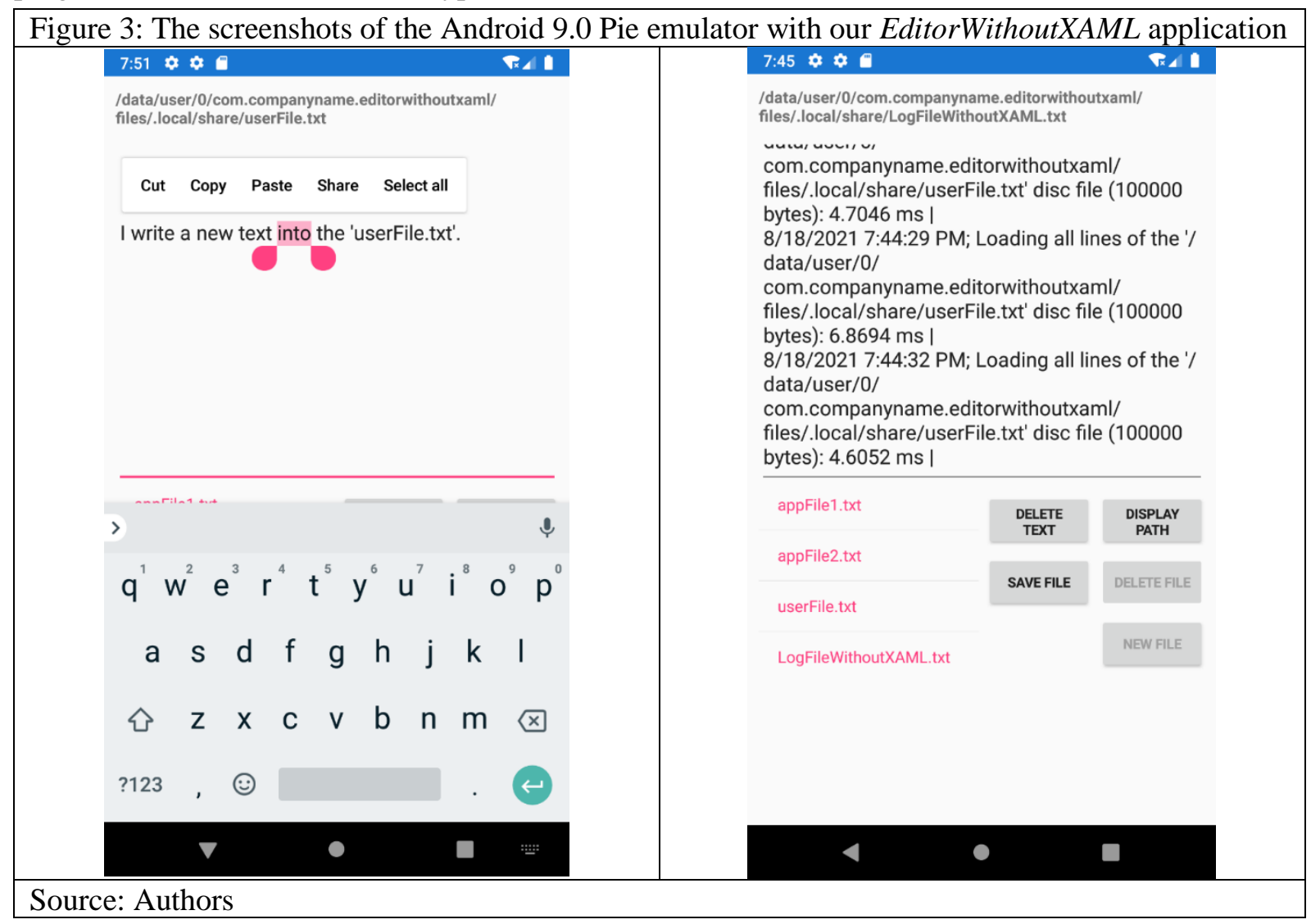

Figure 3 shows screenshots of the Android 9.0 Pie emulator with our EditorWithoutXAML application running on it. This Android Xamarin.Forms application works as a text editor and allows a user to work with the three appFile1.txt, appFile2.txt, and LogFileWithoutXAML.txt disc files. The user can create a new userFile.txt file to work with it and store the file's new content, and then, he/she can also delete this file. The EditorWithoutXAML application provides an Android keyboard and Copy \& Paste tools to 
the user while working with these disc files. Our application also displays the directory path to the currently opened disc file.

\section{A Comparison of the Process of Handling Events in the EditorWithXAML and EditorWithoutXAML Applications}

The processes of handling events in the EditorWithXAML and EditorWithoutXAML applications are quite unalike.

The EditorWithXAML application has all the user interface elements created in the MainPage.xaml XAML file, which means that each element in this file is instantiated with a call to the constructor of the corresponding class, and the resultant object is initialized by setting properties apart from attribute values for this element. The attachment of the EditorTextChanged, OnFileListViewItemSelected, OnDeleteTextButtonClicked, OnDisplayPathButtonClicked, OnSaveButtonClicked, OnDeleteButtonClicked, and OnNewFileButtonClicked event handlers to the TextChanged, ItemSelected, and Clicked events in XAML is performed by setting the event attributes of particular elements. The programmer sees through the instantiation and initialization of the user interface controls' objects and attaches event handlers to the events of these objects, as described previously. In fact, these actions are performed during the application build process when the MainPage.xaml XAML file is parsed and the following MainPage.xaml.g.cs code file is generated by a tool:

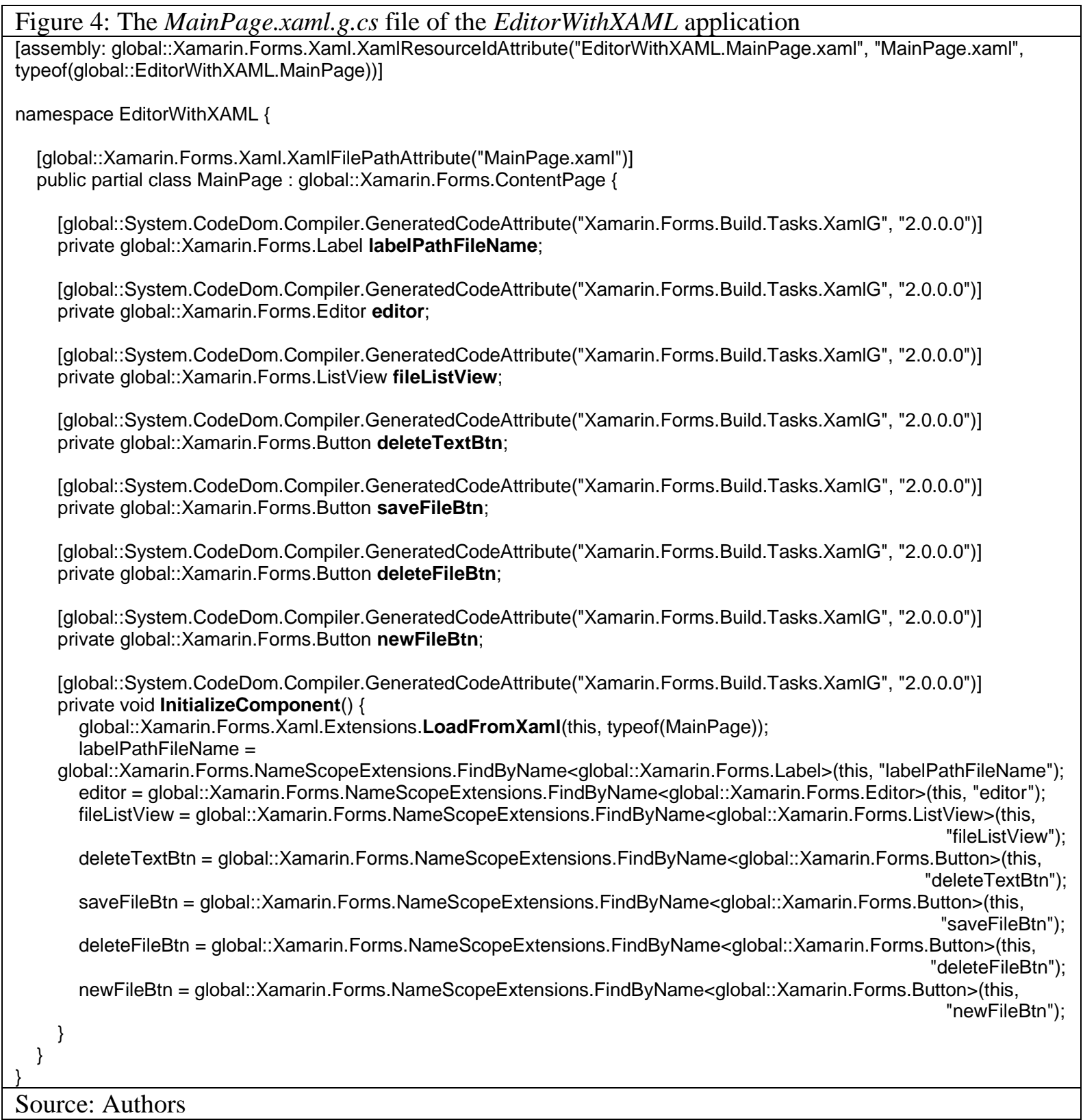


This generated file contains a partial class definition of MainPage which derives from the Xamarin.Forms. ContentPage class and which contains the InitializeComponent method. Right here, in the code of this partial class, we execute real instantiation of the EditorWithXAML application user interface controls' objects. Each element with an $x$ :Name attribute becomes a private object of a given class. The LoadFromXaml method, called by the InitializeComponent method, performs the real initializing the controls' objects and the real attaching the event handlers to the controls' events. Therefore, it initializes all the objects defined in the XAML file, connects them all together in a parentchild relationship, attaches event handlers defined in code to events set in the XAML file, and sets the resultant tree of objects as the content of the page (Microsoft, 2021).

After the MainPage.xaml.g.cs file is generated, this file along with the MainPage.xaml.cs code-behind file are compiled together by the $\mathrm{C \#}$ compiler.

The EditorWithoutXAML application does not use XAML to create controls for its user interface. It instantiates and initializes the objects of these controls directly in the code of its MainPage.xaml.cs file (Figure 2b). Also directly attaching the Editor_TextChanged, FileListView_ItemSelected, DeleteTextbtn_Clicked, DisplayPathbtn_Clicked, SaveFileBtn_Clicked, DeleteFileBtn_Clicked, and NewFileBtn_Clicked event handlers to the TextChanged, ItemSelected, and Clicked events is performed in the code of this file (Figure 2b). Hence, the MainPagexaml.g.cs file generated from the MainPage.xaml file of the EditorWithoutXAML application contains instantiating none objects of controls for its user interface.
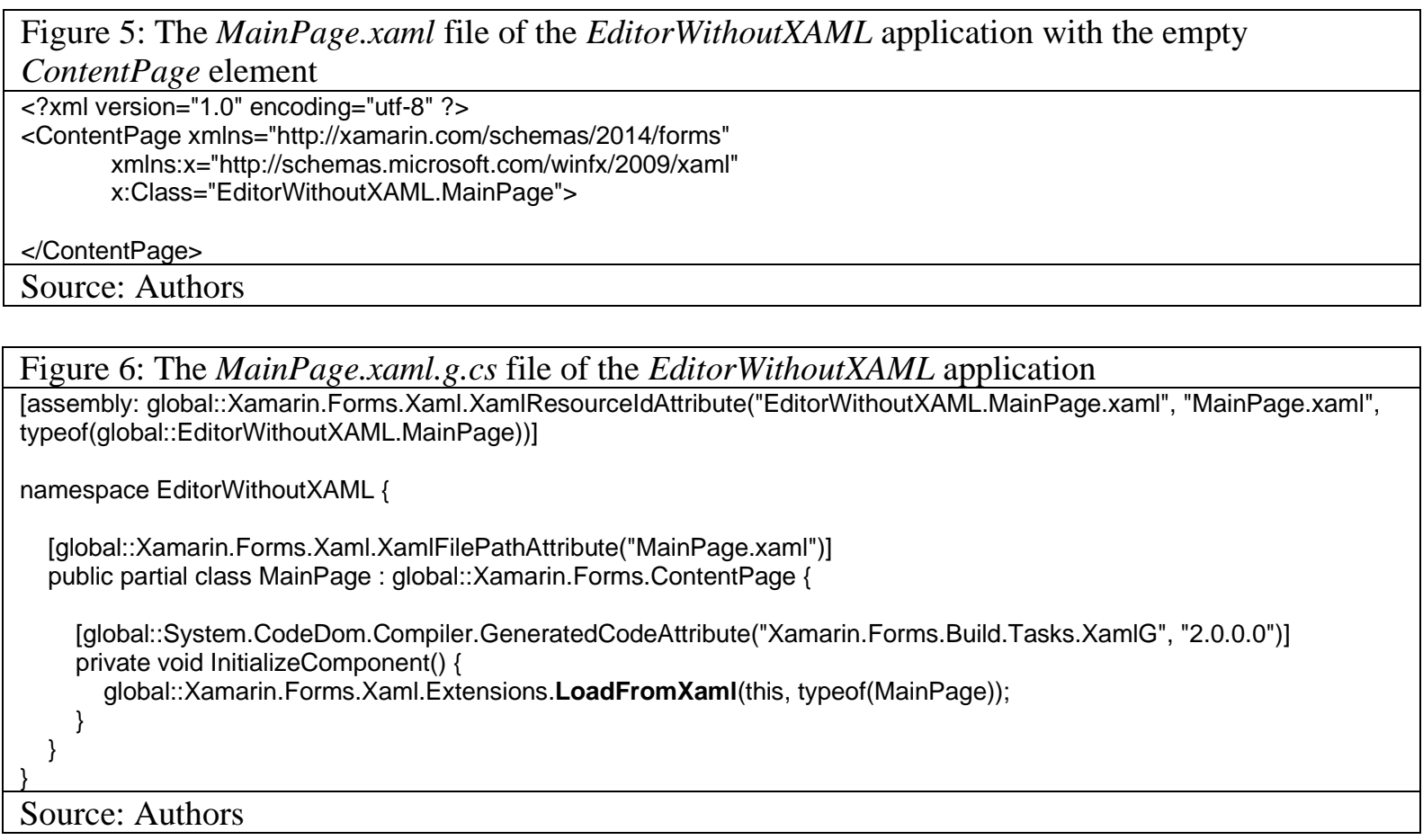

The process of handling events in the EditorWithXAML application that uses XAML to create its user interface controls is much more complex than in the EditorWithoutXAML application that creates its user interface controls directly in the $\mathrm{C \#}$ code. However, this process of handling events is performed by the EditorWithXAML application itself; the programmer does not have to deal with it. If we do not load and do not parse the XAML file of the EditorWithXAML application by calling the LoadFromXaml method at run time (that is the same in our case), then, using XAML to create controls of its user interface does not significantly affect the application's execution efficiency. Besides, it can also provide several benefits to the programmer, e.g., easier modification and maintenance of the user interface XAML code, etc.

\section{The Experiment, its Results and their Brief Analysis}

We assume that the different ways of creating the user interface of Android applications, either in XAML with a C\# support code, or only in C\# code, do not fundamentally affect the execution time of the basic operations with disc files in these applications. 
To verify this assumption, we performed an experiment using the two of our EditorWithXAML and EditorWithoutXAML Android applications. During this experiment, both Android applications performed the loading of data from the userFile.txt disc files into their editor controls and the storing of data from these controls into the userFile.txt disc filesEach of these applications was measured for the execution time of this particular operation, later written into its log file. The EditorWithXAML Android application wrote its execution time into the LogFileWithXAML.txt file, and the EditorWithoutXAML Android application wrote its own execution time into LogFileWithoutXAML.txt. Both operations, loading and storing data, were performed by both the Android applications on the same 10 data sets which contained ASCII characters and had the following sizes: 10000 bytes $(9.77 \mathrm{kB}), 20000$ bytes $(19.53 \mathrm{kB}), 30000$ bytes $(29.30 \mathrm{kB}), 40000$ bytes $(39.06 \mathrm{kB}), 50000$ bytes $(48.83 \mathrm{kB}), 60000$ bytes $(58.59 \mathrm{kB}), 70000$ bytes $(68.36 \mathrm{kB}), 80000$ bytes $(78.13 \mathrm{kB}), 90000$ bytes $(87.89 \mathrm{kB}), 100000$ bytes $(97.66 \mathrm{kB})$. Both the Android applications were running on the Android 9.0 Pie emulator, which in turn ran on the Microsoft Windows 10 Home operating system. The computer on which the experiment was performed had the following basic hardware configuration: Intel Core i5-8250U Processor (6MB Cache, $1.60 \mathrm{GHz}, 4 \mathrm{GT} / \mathrm{s}$, 4 Cores, 8 Threads), RAM: 8 GB.

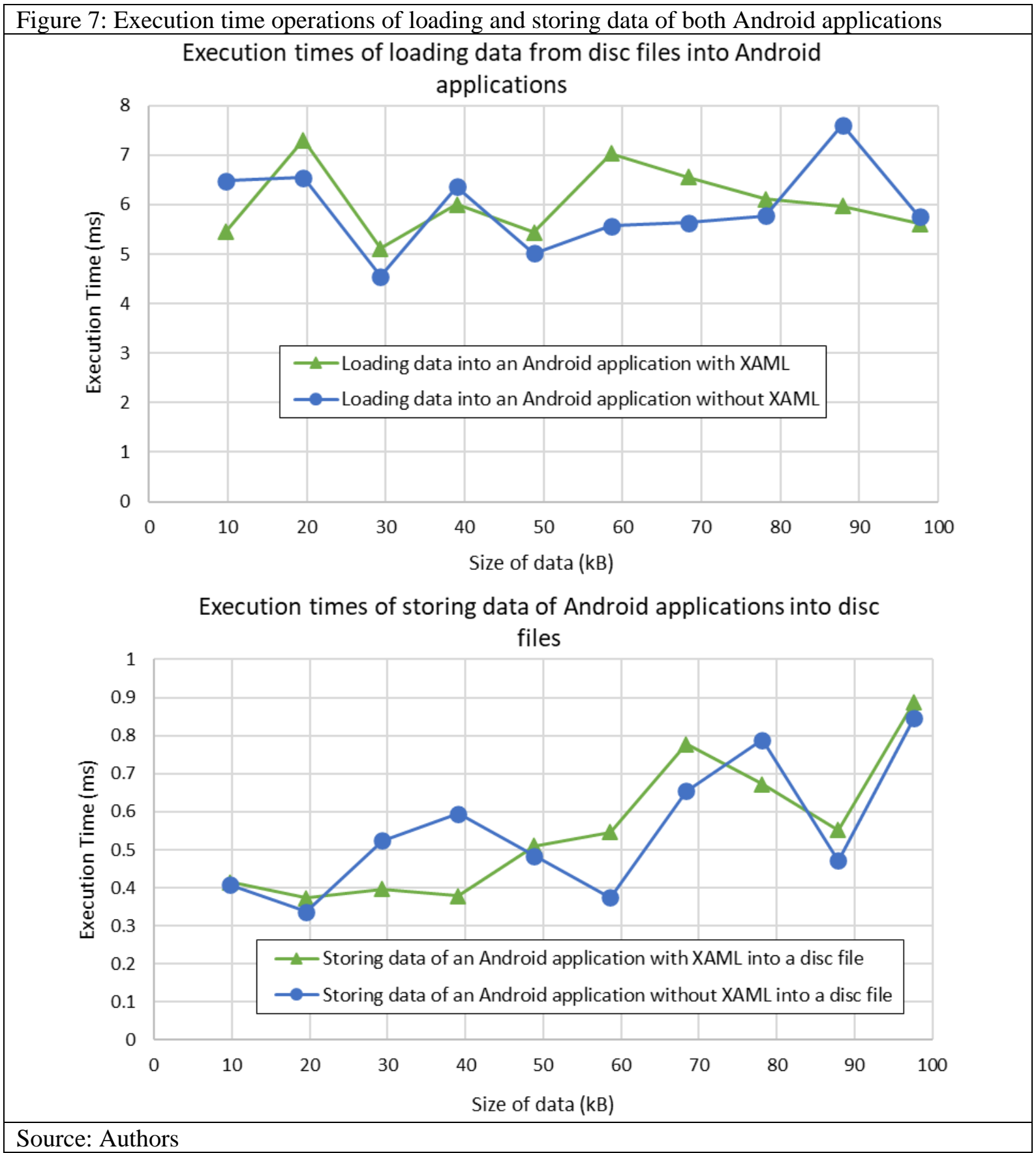


One of the outputs of the EditorWithoutXAML Android application displayed in its editor control, which shows the measured execution times of the operation for loading data from the userFile.txt disc file with a size of 100000 bytes into the editor control, is shown in Figure 3 (the right). Similar outputs were displayed by the EditorWithXAML Android application in its editor control.

The execution time of loading and storing the same 10 data sets by both Android applications are clearly displayed in the graphs (Figure 7).

Brief results analysis

It is obvious from the above graphs which show the execution time of loading and storing the same 10 data sets by both the Android applications that the differences between the execution time of the same operations on the same data by the EditorWithXAML application and EditorWithoutXAML application are minimal. The greatest measured variance in the execution time while loading data between the applications is $1.643 \mathrm{~ms}$, and while storing data, it is $0.2163 \mathrm{~ms}$. These are very small deviations. Thus, it follows that the different ways of creating the user interface of an Android application, whether by XAML with C\# support code or only by C\#, do not fundamentally affect the execution time of the basic operations including disc files that are performed by the application. This confirms our hypothesis. So, the use of XAML to create the user interface of the Android application does not worsen the execution time of the examined basic operations of the Android application.

\section{Conclusion}

It is evident from the comparison of the XAML code and the C\# code for the two of our Android Xamarin.Forms application user interfaces, having the same visual appearance, that the XAML code "provides a more succinct and elegant definition of the user interface and has a visual structure that better mimics the tree organization of the visual elements on the page" (Petzold, 2016) and the XAML code is more concise than the equivalent C\# code (Petzold, 2016). Additionally, we can see from this comparison that it is possible to define all objects of the Android application user interface controls together with the configurations of their properties in the XAML code, and we would only need to write event handlers for these controls in the C\# code. The software company can then divide the work on creating the Android Xamarin.Forms applications user interfaces between designers and programmers. This would allow the company to develop applications with an attractive user interface with a running flawless, efficient, and powerful application core. These facts confirm the hypothesis that we formulated towards the end of the Introduction.

It is evident from our comparison of the process of handling events in EditorWithXAML and EditorWithoutXAML applications that the programmer doesn't have to deal with the process of handling events of controls of a user interface created in the XAML code because this process is already performed by the EditorWithXAML application itself.

From the experiment, it is obvious that the method used to create the Android application user interface (in XAML with C\# support code, or in C\#) does not fundamentally affect the execution time of basic operations with disc files in the application. So, using XAML to create the Android application user interface does not worsen the execution time of some of thebasic operations of the application.

Our findings suggest that it is advantageous to create an Android application user interface in XAML with the C\# support code as it also agrees with the results of this experiment.

\section{Acknowledgements}

This work was supported by project VEGA No. 1/0373/18 entitled "Big data analytics as a tool for increasing the competitiveness of enterprises and supporting informed decisions" by the Ministry of Education, Science, Research and Sport of the Slovak Republic.

\section{References}

Albahari, J., \& Albahari, B. (2017). C\# 7.0 in a Nutshell. O'Reilly Media, Inc.

Butow, E., \& Ryan, T. (2002). C\# Your visual blueprint for building. NET applications. Hungry Minds, Inc.

Microsoft Corporation. (2021, March 8). Documentation. Retrieved from https://docs.microsoft.com

Petzold, Ch. (2016). Creating Mobile Apps with Xamarin.Forms. Xamarin Inc., Redmond: Microsoft Press.

Snider, E. (2018). Mastering Xamarin.Forms. Packt Publishing Limited.

Versluis, G., \& Thewissen, S. (2018). Xamarin.Forms Solutions. APress. 